\title{
Transient growth in linearly stable gravity-driven flow in porous media
}

\author{
G. J. M. Pieters ${ }^{1}$ and C. J. van Duijn \\ Dept. of Mathematics and Computer Science, Eindhoven University of Technology, \\ P.O. Box 513, 5600 MB Eindhoven, The Netherlands
}

\begin{abstract}
In this paper we study gravitational instability of a saline boundary layer formed by evaporation induced upward throughflow at the horizontal surface of a porous medium. Van Duijn et al., [33], derived stability bounds by means of linear stability analysis and an (improved) energy method. These bounds do not coincide, i.e. there exists a subcritical region or stability gap in the system parameter space which is due to the asymmetry of the linear part of the perturbation equations. We show that the linear operator can be symmetrized by means of a similarity transformation. For system parameter values in the stability gap, we show that there exist optimal initial perturbations for which the linearly stable system exhibits transient growth. We show that transient growth is norm dependent by considering weighted norms, which are induced by a one-parameter family of similarity transformations.
\end{abstract}

Key words: Porous media flow; Gravitational instability; Stability analysis; Optimal perturbations; Transient growth.

\section{Introduction}

In this paper we investigate the dynamics of groundwater beneath salt lakes. In particular we study the stability of an equilibrium boundary layer formed by evaporation induced upward throughflow at the horizontal surface of a porous medium. Central issue is to quantify the boundary layer stability or its gravitational instability in terms of the system parameters. This problem was first introduced by [35, 36]. It was later further detailed in [33] where the method of linearised stability and the energy method (with different constraints) were used to establish stability criteria for the boundary layer. Starting point for both methods is a nonlinear perturbation equation which has the abstract form

$\overline{1}$ Corresponding author.

E-mail address: g.j.m.pieters@tue.nl (G.J.M. Pieters) 


$$
\left.\begin{array}{l}
\frac{\mathrm{d} s}{\mathrm{~d} t}=\mathcal{L} s+\mathcal{N}(s) \\
s(t) \in \mathcal{H} \\
s(0)=f^{\star},
\end{array}\right\} t>0,
$$

where $s$ denotes the perturbation with respect to some ground state solution, and where $\mathcal{L}$ represents a linear and $\mathcal{N}$ a nonlinear operator. The boundary conditions are grouped in the solution space $\mathcal{H}$ to which $s$ is said to belong. The energy method uses both $\mathcal{L}$ and $\mathcal{N}$, whereas linear stability analysis is applied to the operator $\mathcal{L}$ only. Both approaches give stability bounds in terms of the system parameters.

The stability bounds found by [33] do not coincide, i.e. there exists a stability gap which was shown to depend on the constraints used in the energy method. One of the first observations regarding the occurence of stability gaps was given by $[2,3,4]$, and, independently and in a different context, by [13, 14] and later by [7]. For a general overview we refer to [6]. These authors showed that one of the essential connections between linear and nonlinear theory is the idea of symmetry of the linear operator $\mathcal{L}$. Therefore we write $\mathcal{L}=\mathcal{L}_{\mathrm{s}}+\mathcal{L}_{\mathrm{a}}$, where $\mathcal{L}_{\mathrm{s}}$ denotes the symmetric and $\mathcal{L}_{\mathrm{a}}$ the asymmetric part of $\mathcal{L}$. In particular, when the nonlinear term satisfies $(\mathcal{N}(s), s) \leqslant 0$, where $(\cdot, \cdot)$ denotes an appropriately chosen inner product, usually the $L^{2}$-inner product, [7] showed that nonlinear stability analysis reduces to the study of the spectrum of the symmetric part of the linear operator. Hence, when $\mathcal{L}$ is symmetric, both methods give similar stability bounds.

The presence of throughflow in the salt-lake problem results in a first-order term in the stability equation implying $\mathcal{L}_{\mathrm{a}} \neq 0$. Hence the spectrum of $\mathcal{L}$ does not coincide with the spectrum of $\mathcal{L}_{\mathrm{s}}$, and this results in a stability gap.

To quantify this gap and to understand the behaviour of the physical system in this - so called - subcritical region, a modified energy method is needed [7]. One method is to formulate a "generalized energy" by coupling different norms with suitable coupling parameters, see [31]. The idea is to optimize these coupling parameters to obtain a sharper energy stability bound. In some cases it even removes the stability gap. Such energies are introduced on purely heuristic grounds.

An alternative approach, which is followed in this paper, is to use the concept of symmetrizable operators. By this we mean that $\mathcal{L}$ may be asymmetric with respect to one inner product, but symmetric with respect to another. This can usually be achieved by reformulating the original problem in terms of a newly defined quantity. For example, such a new quantity may be equal to the original quantity multiplied by a weight function. A sufficient condition for which an operator $\mathcal{L}$ is symmetrizable, is that there exists an invertible operator $\mathcal{M}$ such that $\mathcal{M}^{-1} \mathcal{L} \mathcal{M}$ is symmetric. Such an operator defines a similarity transformation and the resulting operator $\mathcal{M}^{-1} \mathcal{L} \mathcal{M}$ is called similar (to $\mathcal{L}$ ). Besides being symmetric, a similar operator has the important property that its spectrum is a subset of the spectrum of $\mathcal{L}$. Once we have found such an operator $\mathcal{M}$, we can apply [7]: due to the symmetry of the similar operator, the linear stability bound coincides with the nonlinear 
stability bound provided that the nonlinear term satisfies $(\mathcal{N}(s), s)_{\mathcal{M}} \leqslant 0$, where $(\cdot, \cdot)_{\mathcal{M}}:=\left(\mathcal{M}^{-1}(\cdot), \mathcal{M}^{-1}(\cdot)\right)$. Here nonlinear stability is measured in the energy norm induced by $(\cdot, \cdot)_{\mathcal{M}}$. For other applications of similarity transformations we refer to [6, Sections 4 and 5]. The use of transformations and the consequences for the stability analysis is also discussed in [32].

The introduction of different inner-products affects the orthogonality of the eigenfunctions of the (asymmetric) operator $\mathcal{L}$ : for instance, the eigenfunctions may be non-orthogonal with respect to the $L^{2}$ inner-product, but orthogonal with respect to a weighted $L^{2}$ inner-product. This results from the fact that the operator is nonnormal [16]. Due to the non-orthogonality of the eigenfunctions, nonnormal operators exhibit particular transient behaviour which cannot be captured by linear stability analysis. In other words, the linearly stable but nonnormal system may temporarily move far away from equilibrium before approaching it as $t \rightarrow \infty$, while undergoing a considerable (transient) algebraic growth of the energy norm. This is studied by [25] and later by [5]. The concept is that a group of eigenfunctions are nearly linearly dependent so that particular initial disturbances may involve large coefficients.

With this mechanism in mind, one could determine the optimal initial condition that produces the largest relative energy growth during a certain time period. This is done by [25]. Their variational optimization method relies on the fact that every initial perturbation can be decomposed into eigenfunctions, i.e. the eigenfunctions form a complete set. For certain problems on semi-infinite domains, however, like the Blasius boundary layer [10, 27], one can show that there is a finite number of eigenfunctions corresponding to a discrete point spectrum and that there are solutions that correspond to an uncountable point spectrum. The latter is sometimes referred to as the continuum. In this paper we consider a saline boundary layer in a semi-infinite domain for which both spectra occur. It is clear the one can only determine a finite number of discrete eigenvalues and corresponding eigenfunctions. Hence the determination of the optimal initial perturbation via a variational optimization procedure is limited since one should have to incorporate the remaining part of the spectrum, see also [18].

However, it is still possible to find initial perturbations that initiate transient growth. These special perturbations can be determined by analysing the numerical range [16] of the operator $\mathcal{L}$. The numerical range is the largest eigenvalue of the operator $\mathcal{L}_{\mathrm{s}}$ and this eigenvalue is equal to the initial slope of the energy norm ([5]).

This interpretation has led to general analysis of nonnormal systems that has since been used extensively to understand transient growth in deformation and shear flows. For further references see, for example, [28] and [1].

This paper is organized as follows. In Section 2, we formulate the porous medium flow problem and we introduce the equilibrium saline boundary layer as the ground state solution.

In Section 3 we analyse the spectrum of the linear operator; i.e. we study the linearised perturbation equations. We also recall some earlier results [33] about the stability bounds: a stability bound resulting from the linear theory and a smaller 
bound resulting from from an energy method. Below the latter bound all perturbations decay in $L^{2}$-sense with respect to time.

In Sections 4 and 5 we consider the behaviour of the linearised perturbation equations for parameter values between these stability bounds. In Section 4 we demonstrate transient growth of the $L^{2}$-norm of perturbations and we construct some bounds on this growth. In Section 5 we show that transient growth is normdependent. In particular we introduce a one-parameter family of transformations that imply weighted $L^{2}$-norms. Dependent on the parameter value, transient growth may still occur or disappear.

At various parts of this paper we present numerical results about spectra and eigenfunctions. For this we used a modified Chebyshev-Galerkin method. Details are outlined in the appendix. Conclusions are summarized in Section 6.

\section{Problem formulation}

Following [33], we consider a homogeneous and uniform isotropic porous medium occupying the three-dimensional halfspace

$$
\Omega=\left\{(x, y, z):(x, y) \in \mathbb{R}^{2}, z>0\right\} .
$$

The dimensionless equations in terms of the Boussinesq approximation are given by $([35,20])$ :

- Fluid incompressibility

$$
\operatorname{div} \mathbf{U}=0
$$

- Darcy's law

$$
\mathbf{U}+\operatorname{grad} P-S \mathbf{e}_{z}=\mathbf{0} ;
$$

- Salt transport

$$
\frac{\partial S}{\partial t}+R_{S} \mathbf{U} \cdot \operatorname{grad} S=\operatorname{div} \operatorname{grad} S
$$

in $\Omega$ and for all $t>0$, subject to the boundary conditions

$$
\mathbf{U} \cdot \mathbf{e}_{z}=-R_{S}^{-1} \quad \text { and } \quad S=1 \quad \text { at } z=0 \text {, for all } t>0 \text {. }
$$

Here $S$ denotes salt saturation, $\mathbf{U}$ fluid velocity and $P$ an appropriately chosen dimensionless pressure. Further, $\mathbf{e}_{z}$ denotes the unit vector in $z$-direction, pointing downwards. The system Rayleigh number $R_{S}$ is given by

$$
R_{s}=\frac{\left(\rho_{m}-\rho_{r}\right) g \kappa}{\mu E}
$$


where $\mu$ is fluid viscosity, $\kappa$ medium permeability, $g$ gravity constant, $E$ evaporation rate, $\rho_{m}$ maximum fluid density at the outflow boundary, and $\rho_{r}$ fluid density in 'natural circumstances' (i.e. far away from the outflow boundary).

To obtain a unique solution satisfying equations (2) and boundary conditions (3), an initial condition is needed:

$$
\left.S\right|_{t=0}=f^{\star} \quad \text { in } \Omega
$$

where $f^{\star}$ denotes the - as yet - unspecified salt saturation at the initial time $t=0$. Remark 1 Note that we no not impose boundary conditions at $z=\infty$. The behaviour at $z=\infty$ is implied by the initial condition $f^{\star}$ and by physically plausible growth conditions for the solutions. This is explained in [21].

\subsection{The ground state solution}

In this paper we are concerned with the stability of the time-independent solution of (2) and (3) that vanishes at large depth, i.e. as $z \rightarrow \infty$. This solution is called the ground state. It represents an equilibrium boundary layer below the surface $z=0$, which is sustained by evaporation induced throughflow. It is explicitly given by the uniform upflow

$$
\mathbf{U}=\mathbf{U}_{0}:=-R_{s}^{-1} \mathbf{e}_{z} \quad \text { in } \Omega,
$$

and by the salt saturation $S=S_{0}(z)$ satisfying

$$
\begin{cases}\frac{\partial^{2} S_{0}}{\partial z^{2}}+\frac{\partial S_{0}}{\partial z}=0, & \text { for } z>0, \\ S_{0}(0)=1, & S_{0}(\infty)=0 .\end{cases}
$$

Clearly,

$$
S_{0}(z)=\mathrm{e}^{-z}, \quad z>0
$$

The corresponding ground state pressure $P=P_{0}$ is found by integrating Darcy's law (2b).

\subsection{Perturbation equations}

The stability analysis is based on the expansion

$$
S=S_{0}+s, \quad \mathbf{U}=\mathbf{U}_{0}+\mathbf{u}, \quad P=P_{0}+p,
$$


where $\mathbf{u}=(u, v, w)$. Substituting (8) into equations (2) and writing $R$ instead of $R_{s}$, yields for the perturbations the system (in $\Omega$ and for all $t>0$ )

$$
\begin{array}{r}
\operatorname{div} \mathbf{u}=0, \\
\mathbf{u}+\operatorname{grad} p-s \mathbf{e}_{z}=\mathbf{0}, \\
\frac{\partial s}{\partial t}-\frac{\partial s}{\partial z}+R \frac{\partial S_{0}}{\partial z} w+R \mathbf{u} \cdot \operatorname{grad} s=\Delta s,
\end{array}
$$

and the homogeneous boundary conditions

$$
s=\mathbf{u} \cdot \mathbf{e}_{z}=w=0 \quad \text { at } z=0, \text { for all } t>0 .
$$

As in [17] we note that equations (9a) and (9b) can be combined to give for $s$ and $w$ the linear relation

$$
\Delta w=\Delta_{\perp} S \quad \text { in } \Omega .
$$

Here $\Delta_{\perp}$ denotes the horizontal Laplacian $\partial_{x x}+\partial_{y y}$.

\section{Linear stability of the equilibrium boundary layer}

The stability of the ground state (6), (7) was investigated by [33]. They used linear stability theory, i.e. disregarding the product term $R \mathbf{u} \cdot \operatorname{grad} s$ in equation (9c), and an improved energy method using equation (11) as pointwise constraint. Restricted to periodic perturbations of the form

$$
\{s, w\}(x, y, z, t)=\{s, w\}(z, t) \mathrm{e}^{i\left(a_{x} x+a_{y} y\right)},
$$

where $a_{x}$ and $a_{y}$ denote the horizontal wavenumbers, they obtained two stability bounds. With $a^{2}=a_{x}^{2}+a_{y}^{2}$, these bounds are represented in the $(a, R)$-plane by the curves

$$
0<R_{E}(a)<R_{L}(a) \quad \text { for } a>0 .
$$

The ground state is unstable above the curve $R_{L}(a)$, and definitely stable below the curve $R_{E}(a)$ in the sense that the $L^{2}$-norm of any periodic perturbation decays in time when $0<a<\infty$ and $0<R<R_{E}(a)$. The two bounds are shown in Figure 1. The main goal of this paper is to understand the behaviour of $(x, y)$-periodic solutions of the linearized perturbation equations for points $(a, R)$ in between these stability bounds. We start with some obervations concerning the spectrum and the corresponding eigenfunctions.

\subsection{Properties of the spectrum of the linear operator}

We consider the linear part of (9c) and (11) in $Q:=\{(z, t): z>0, t>0\}$. Assuming $f^{\star}=f^{\star}(z)$ and periodicity of $s$ and $w$ with respect to the horizontal 


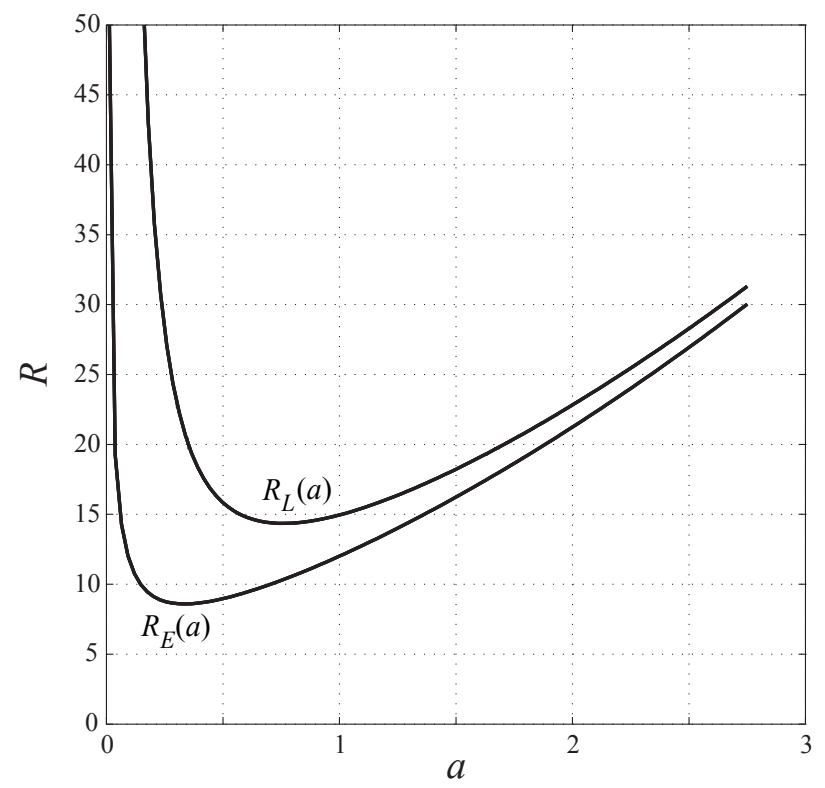

Fig. 1. Stability bounds involving the lowest eigenvalue $R=R_{\min }$ versus wavenumber $a$ : top curve $R=R_{L}(a)$ results from the linear stability method, bottom curve $R=R_{E}(a)$ results from the energy method.

plane, as in (12), we obtain the equations

$$
(\mathrm{LP}) \begin{cases}\frac{\partial s}{\partial t}=D^{2} s+D s-a^{2} s+R \mathrm{e}^{-z} B(s)=: L s & \text { in } Q, \\ B^{-1}(w):=-a^{-2} D^{2} w+w=s & \text { in } \mathbb{R}_{+} \text {and for each } t>0, \\ w(0, t)=s(0, t)=0 & \text { for all } t>0, \\ s(z, 0)=f(z) & \text { for all } z>0 .\end{cases}
$$

In (LP) we used the notation $D:=\partial_{z}$. Because $s$ is the perturbation of (7) we have $f(z)=f^{\star}(z)-\mathrm{e}^{-z}$. Note that we do not impose the boundary condition $w(\infty)=0$ to solve the $w$-problem. This is a consequence of the fact that $s(\cdot, t) \in L^{2}\left(\mathbb{R}_{+}\right)$, see [21] for details. To investigate the spectrum of $L$, we fix $a>0$ and $R>0$ and consider the eigenvalue problem

$$
L s=D^{2} s+D s-a^{2} s+R \mathrm{e}^{-z} B(s)=\sigma s, \quad s \in H_{0}^{1}\left(\mathbb{R}_{+}\right) .
$$

Here $H_{0}^{1}\left(\mathbb{R}_{+}\right)$denotes the Hilbert space of square integrable functions having a square integrable derivative and vanishing at $z=0$. Since no explicit solutions of (14) are known, we solved the eigenvalue problem numerically by means of a modified Chebyshev-Galerkin method. Details of this method are given in the appendix. Later on in this section we show by means of a similarity transformation that the discrete eigenvalues $\left\{\sigma_{i}=\sigma_{i}(a, R)\right\}$ of (14) are real. Let them be ordered 

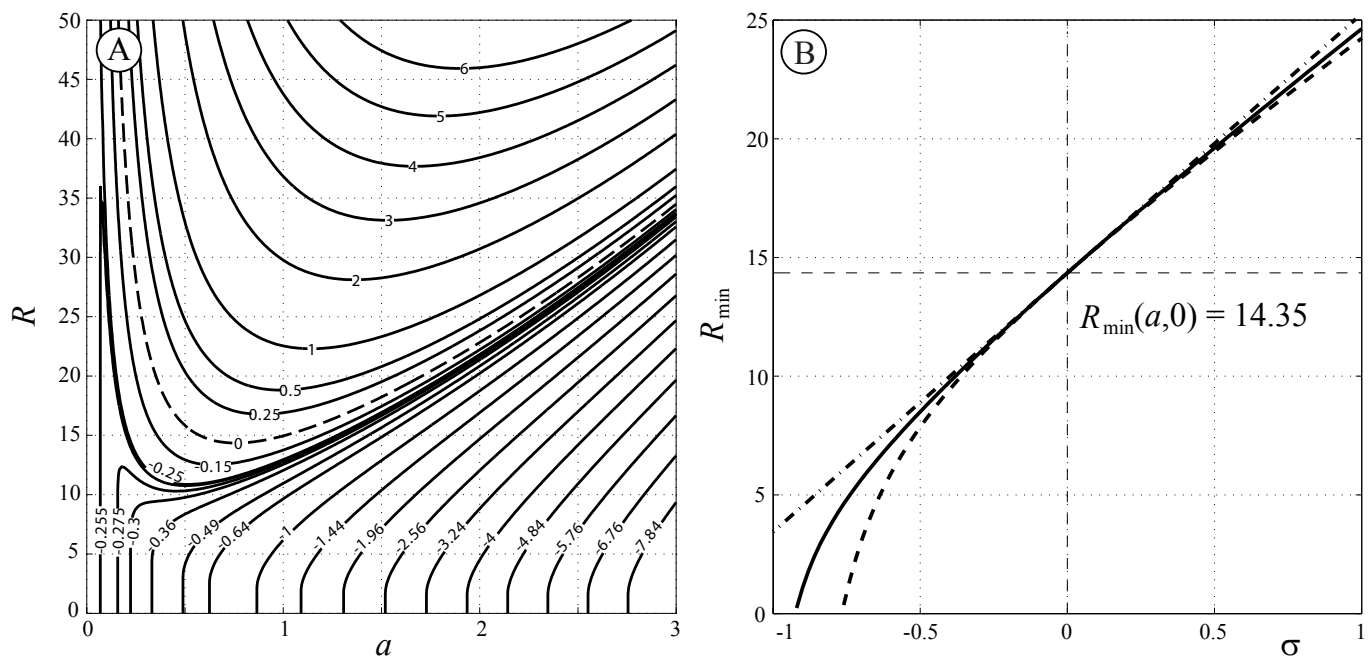

Fig. 2. (A) Isocurves of the largest approximate eigenvalue $\sigma_{\max }$ of operator $L$. The dashed curve $\sigma_{\max } \equiv 0$ corresponds to the neutral stability curve $R_{L}(a)$ (see text for its definition). (B) Upper and lower bounds of $R_{\min }(a, \sigma)$ for $a=0.759$ as function of the growthrate $\sigma$.

according to

$$
\cdots<\sigma_{n}<\cdots<\sigma_{2}<\sigma_{1}=: \sigma_{\max }
$$

For $a$ and $R$ in relevant ranges, Figure 2 shows numerically obtained isocurves of $\sigma_{\max }$. Putting $R=0$ in (14) gives the explicit expression $\sigma_{\max }=-a^{2}-\frac{1}{4}$. This is recovered numerically. Further observe that the isocurves become vertical as $R \searrow 0$.

The dashed isocurve (corresponding to $\sigma_{\max } \equiv 0$ ) in Figure 2 (left) is usually referred to as the neutral stability curve. It is often found by solving the rewritten eigenvalue problem

$$
\mathscr{L}_{\sigma} s:=D^{2} s+D s-\left(a^{2}+\sigma\right) s=-\operatorname{Re}^{-z} B(s)=: R \mathscr{B} s
$$

with $s \in H_{0}^{1}\left(\mathbb{R}_{+}\right)$, see [33]. In other words, the role of $\sigma$ and $R$ as eigenvalues can be interchanged. Suppose again that the set of eigenvalues $\left\{R_{i}=R_{i}(a, \sigma)\right\}$ of problem (15) are ordered by $R_{\min }:=R_{1}<R_{2}<\cdots<R_{n}<\cdots$. Then the neutral stability curve is defined by $R_{L}(a):=R_{\min }(a, \sigma=0)$. It is also shown as the top curve in Figure 1.

In the sequel of this section we investigate the behaviour of $R_{\min }(a, \sigma)$ near $\sigma=0$. Figure 2 suggests that for fixed $a>0$, at least for $\sigma$ sufficiently close to zero, we have $\sigma \gtrless 0$ if and only if $R \gtrless R_{\min }(a, \sigma=0)=R_{L}(a)$. This property is crucial in linearised stability theory. Its physical interpretation is that the conductive ground state exchanges stability with a convective flow regime. In general, exchange of stability is straightforward to prove when the linear operator $L$ is symmetric with respect to $L^{2}\left(\mathbb{R}_{+}\right)$, see [7]. However, the operator $L$ (and $\mathscr{L}_{\sigma}$ ) is asymmetric in $L^{2}\left(\mathbb{R}_{+}\right)$due to the occurence of the first order derivative. To show the stability exchange, we will make use of a particular (similarity) transformation 
which symmetrizes the operator $L$ in $L^{2}\left(\mathbb{R}_{+}\right)$. Following [26, Section 5], we set

$$
s(z, t)=M u(z, t):=\mathrm{e}^{-\frac{1}{2} z} u(z, t) .
$$

Then

$$
L s=\mathrm{e}^{-\frac{1}{2} z}\left[D^{2} u+\left(-\frac{1}{4}-a^{2}\right) u+R \mathrm{e}^{\left(-\frac{1}{2} z\right.} B\left(\mathrm{e}^{-\frac{1}{2} z} u\right)\right]=: M \widehat{L} M^{-1} s
$$

where $\widehat{L}$ is given by

$$
\widehat{L}=D^{2}+\left(-\frac{1}{4}-a^{2}\right) I+R \mathrm{e}^{-\frac{1}{2} z} B\left(\mathrm{e}^{-\frac{1}{2} z} \cdot\right),
$$

in which $I$ denotes the identity operator. Now we consider the problem

$$
(\widehat{\mathrm{LP}})\left\{\begin{array}{l}
\frac{\partial u}{\partial t}=D^{2} u+\left(-\frac{1}{4}-a^{2}\right) u+R \mathrm{e}^{-\frac{1}{2} z} B\left(\mathrm{e}^{-\frac{1}{2} z} u\right)=\widehat{L} u \\
B^{-1}(w)=-a^{-2} D^{2} w+w=\mathrm{e}^{-\frac{1}{2} z} u
\end{array}\right.
$$

in $Q$, with

$$
w(0, t)=u(0, t)=0 \quad \text { for all } t>0
$$

and

$$
u(z, 0)=g(z):=\mathrm{e}^{\frac{1}{2} z} f(z) \quad \text { for all } z>0 .
$$

Note that the operator $\widehat{L}$ is symmetric with respect to $L^{2}\left(\mathbb{R}_{+}\right)$. Clearly, the spectrum of $\widehat{L}$ is contained in the spectrum of $L$. In fact, the part of the spectrum of $L$ for which the eigenfunctions belong to $\left\{s \in L^{2}\left(\mathbb{R}_{+}\right): s \mathrm{e}^{\frac{1}{2} z} \in L^{2}\left(\mathbb{R}_{+}\right)\right\}$coincides with the spectrum of $\widehat{L}$ with respect to $L^{2}\left(\mathbb{R}_{+}\right)$. We are now in a position to prove exchange of stability quite easily by using $\widehat{L}$ instead of $L$. For this purpose we rewrite the eigenvalue problem $\widehat{L} u=\sigma u$, as in (15), in the form

$$
\widehat{\mathscr{L}}_{\sigma} u:=D^{2} u-\left(a^{2}+\frac{1}{4}+\sigma\right) u=R \widehat{\mathscr{B}} u
$$

with $\widehat{\mathscr{B}} u:=-\mathrm{e}^{-\frac{1}{2} z} B\left(\mathrm{e}^{-\frac{1}{2} z} u\right)$. We prove

Theorem 2 Let the smallest eigenvalue of (21) be denoted by $R_{\min }(a, \sigma)$ and suppose that it depends smoothly on $a>0$ and $\sigma>-a^{2}-\frac{1}{4}$. Then there exist a positive constant $c$ and a smooth function $h:\left(-a^{2}-\frac{1}{4}, \infty\right) \longmapsto \mathbb{R}$, satisfying $h(0)=0$, $h(\sigma) \gtrless 0$ if $\sigma \gtrless 0$ and $h(\sigma) \leqslant$ c $\sigma$ for all $\sigma>-a^{2}-\frac{1}{4}$, such that

$$
h(\sigma) \leqslant R_{\min }(a, \sigma)-R_{L}(a) \leqslant c \sigma .
$$

Moreover, $h^{\prime \prime}(0)<0$. 
PROOF. First observe that

$$
(\widehat{\mathscr{B}} u, u)=-\left(B\left(\mathrm{e}^{-\frac{1}{2} z} u\right), \mathrm{e}^{-\frac{1}{2} z} u\right)=-(w, s),
$$

and $(w, s)=a^{-2}\|D w\|_{2}^{2}+\|w\|_{2}^{2}>0$. Here $(\cdot, \cdot)$ denotes the usual $L^{2}\left(\mathbb{R}_{+}\right)$inner product. Hence $(\mathscr{B} u, u)<0$. Further, $\mathscr{L}_{\sigma}=\mathscr{L}_{0}-\sigma I$ where $I$ denotes the identity operator. Since $\mathscr{L}_{\sigma}$ and $\mathscr{B}$ are self-adjoint, we have the following variational characterization of $R_{\min }(a, \sigma)$ :

$$
0<R_{\min }(a, \sigma)=\inf _{\bar{u} \in H_{0}^{1}\left(\mathbb{R}_{+}\right)} \frac{(\widehat{\mathscr{L}} \bar{u}, \bar{u})}{(\widehat{\mathscr{B}} \bar{u}, \bar{u})}=\frac{\left(\widehat{\mathscr{L}} u_{\sigma}, u_{\sigma}\right)}{\left(\widehat{\mathscr{B}} u_{\sigma}, u_{\sigma}\right)}
$$

In particular, for $\sigma=0$, we have

$$
\begin{aligned}
0<R_{\min }(a, 0) & =\inf _{\bar{u} \in H_{0}^{1}\left(\mathbb{R}_{+}\right)} \frac{(\widehat{\mathscr{L}} \bar{u}, \bar{u})}{(\widehat{\mathscr{B}} \bar{u}, \bar{u})} \leqslant \frac{\left(\widehat{\mathscr{L}} u_{\sigma}, u_{\sigma}\right)}{\left(\widehat{\mathscr{B}} u_{\sigma}, u_{\sigma}\right)}= \\
& =\frac{\left(\widehat{\mathscr{L}} u_{\sigma}, u_{\sigma}\right)}{\left(\widehat{\mathscr{B}} u_{\sigma}, u_{\sigma}\right)}+\sigma \frac{\left(u_{\sigma}, u_{\sigma}\right)}{\left(\widehat{\mathscr{B}} u_{\sigma}, u_{\sigma}\right)}=R_{\min }(a, \sigma)+\sigma \frac{\left\|u_{\sigma}\right\|_{2}^{2}}{\left(\widehat{\mathscr{B}} u_{\sigma}, u_{\sigma}\right)} .
\end{aligned}
$$

This implies

$$
R_{\min }(a, \sigma)-R_{L}(a) \geqslant \sigma \frac{\left\|u_{\sigma}\right\|_{2}^{2}}{-\left(\widehat{\mathscr{B}} u_{\sigma}, u_{\sigma}\right)}=: h(\sigma) .
$$

In a similar way we find the upper bound

$$
R_{\min }(a, \sigma)-R_{L}(a) \leqslant \frac{\left\|u_{0}\right\|_{2}^{2}}{-\left(\widehat{\mathscr{B}} u_{0}, u_{0}\right)} \sigma=: c \sigma .
$$

This proves (22) and in particular $R_{\min }(a, \sigma) \gtrless R_{L}(a)$ for $\sigma \gtrless 0$. Because $\left(\widehat{\mathscr{B}} u_{0}, u_{0}\right)<$ 0 , inequalities (24) and (25) also imply

$$
0<\frac{\left\|u_{0}\right\|_{2}^{2}}{-\left(\widehat{\mathscr{B}} u_{0}, u_{0}\right)}=\left.\frac{\partial R_{\min }(a, \sigma)}{\partial \sigma}\right|_{\sigma=0} .
$$

Differentiating $\widehat{\mathscr{L}_{\sigma}} u_{\sigma}=R_{\min }(a, \sigma) \widehat{\mathscr{B}} u_{\sigma}$ with respect to $\sigma$ and setting $v_{\sigma}=\mathrm{d} u_{\sigma} / \mathrm{d} \sigma$ gives

$$
\widehat{\mathscr{L}}_{\sigma} v_{\sigma}-u_{\sigma}=R_{\min }(a, \sigma) \widehat{\mathscr{B}} v_{\sigma}+\frac{\partial R_{\min }(a, \sigma)}{\partial \sigma} \widehat{\mathscr{B}} u_{\sigma}
$$

implying

$$
\left(\widehat{\mathscr{L}}_{\sigma} v_{\sigma}-R_{\min }(a, \sigma) \widehat{\mathscr{B}} v_{\sigma}, v_{\sigma}\right)-\left(u_{\sigma}, v_{\sigma}\right)=\frac{\partial R_{\min }(a, \sigma)}{\partial \sigma}\left(\widehat{\mathscr{B}} u_{\sigma}, v_{\sigma}\right)
$$


Since $\widehat{\mathscr{B}}$ is self-adjoint in $L^{2}\left(\mathbb{R}_{+}\right)$, we have

$$
\begin{aligned}
& \left(u_{\sigma}, v_{\sigma}\right)=\frac{1}{2} \frac{\mathrm{d}}{\mathrm{d} \sigma}\left\|u_{\sigma}\right\|_{2}^{2}, \quad\left(\widehat{\mathscr{B}} u_{\sigma}, v_{\sigma}\right)=\frac{1}{2} \frac{\mathrm{d}}{\mathrm{d} \sigma}\left(\widehat{\mathscr{B}} u_{\sigma}, u_{\sigma}\right), \\
& \left(\widehat{\mathscr{L}_{\sigma}} v_{\sigma}-R_{\min }(a, \sigma) \widehat{\mathscr{B}} v_{\sigma}, v_{\sigma}\right)<0,
\end{aligned}
$$

we thus find

$$
\frac{\mathrm{d}}{\mathrm{d} \sigma}\left\|u_{\sigma}\right\|_{2}^{2}<-\frac{\partial R_{\min }(a, \sigma)}{\partial \sigma} \frac{\mathrm{d}}{\mathrm{d} \sigma}\left(\widehat{\mathscr{B}} u_{\sigma}, u_{\sigma}\right) .
$$

Next we define $\tilde{h}(\sigma):=\frac{\left\|u_{\sigma}\right\|_{2}^{2}}{-\left(\widehat{\mathscr{B}} u_{\sigma}, u_{\sigma}\right)}$ such that $h(\sigma)=\sigma \widetilde{h}(\sigma)$. Differentiating $\tilde{h}(\sigma)$ and using (26) and (27) results in

$$
-\left.\left(\widehat{\mathscr{B}} u_{0}, u_{0}\right) \frac{\mathrm{d}}{\mathrm{d} \sigma}\left\|u_{\sigma}\right\|_{2}^{2}\right|_{\sigma=0}+\left.\left\|u_{0}\right\|_{2}^{2} \frac{\mathrm{d}}{\mathrm{d} \sigma}\left(\widehat{\mathscr{B}} u_{\sigma}, u_{\sigma}\right)\right|_{\sigma=0}<0,
$$

which immediately implies $\widetilde{h}^{\prime}(0)<0$. Since $h^{\prime \prime}(\sigma)=2 \widetilde{h}^{\prime}(\sigma)+\sigma \widetilde{h}^{\prime \prime}(\sigma)$, we find $h^{\prime \prime}(0)<0$ which proves the second assertion of the theorem.

The function $R_{\min }(a, \sigma)$ for $a=0.759$ together with the upper and lower bounds are shown in Figure 2 (right). Theorem 2 implies the following. Let the system Rayleigh number $R_{s}$ be sufficiently close to $R_{L}(a)$. If $R_{s}>R_{L}(a)$, then there exists a $\sigma>0$ such that $R_{s}=R_{\min }(a, \sigma)$. In other words, if $R_{s}>R_{L}(a)$, there exists an infinitesimal perturbation which will grow in time, implying that the boundary layer is unstable. If $R_{S}<R_{L}(a)$ no definite statement about stability can be made. Only certain infinitesimal perturbations now decay. Others, and in particular large perturbations, may still grow in time.

\section{Transient growth behaviour}

In this section we investigate the previous statement further. Employing the energy method, one seeks for conditions in terms of the system parameters $(a, R)$ for which a suitable norm - mostly the $L^{2}$-norm - decays in time. In [33] we showed that if $a>0$ and $R<R_{E}(a)$, see also Figure 1, then solutions of the linear problem (LP) satisfy

$$
\frac{\mathrm{d}}{\mathrm{d} t} \int_{\mathbb{R}_{+}} s^{2}(z, t) \mathrm{d} z<0 \quad \text { for all } t>0,
$$

provided $f \in H_{0}^{1}\left(\mathbb{R}_{+}\right)$. Moreover, this statement holds for periodic solutions of the original nonlinear perturbation equations (9).

To exploit the behaviour when $R_{E}(a)<R<R_{L}(a)$, we first introduce some notation. 

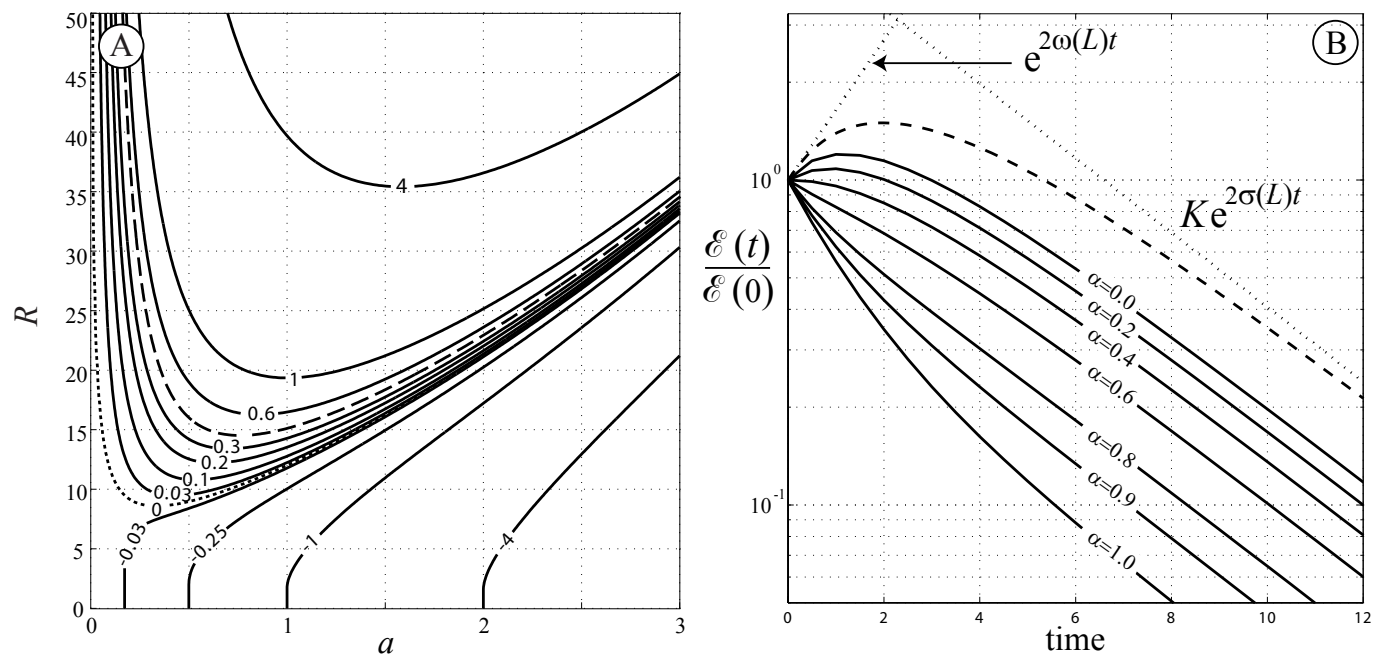

Fig. 3. (A) Isocurves of the largest eigenvalue $\omega_{\max }$ of (31). Dashed curve corresponds to $R_{L}(a)$ and dotted curve to $R_{E}(a)$ (= zero level curve). (B) Transient growth for $a=0.38$ and $R=15$ (in $\mathrm{G}$ ) of the scaled 'energy'. For the dashed curve the initial condition is given by $f=\tilde{f}$ (see (LP)). The initial perturbation for the solid curves is given by a specific $f$ for which $\mathrm{e}^{\frac{1}{2} z} f \in L^{2}\left(\mathbb{R}_{+}\right)$. The upper bound (34) is shown as the composition of the dotted lines.

Definition 3 Let $s(z, t)$ be the solution of problem (LP) with $f \in L^{2}\left(\mathbb{R}_{+}\right)$and let

$$
\mathscr{E}(t):=\int_{\mathbb{R}_{+}} s^{2}(z, t) \mathrm{d} z=:\|s(t)\|_{2}^{2}
$$

(i) The spectral bound of the operator $L$ is defined by

$$
\sigma(L)=\sup _{\lambda \in \Sigma(L)} \operatorname{Re} \lambda
$$

where $\Sigma(L)$ denotes the spectrum of $L$.

(ii) The numerical range of the operator $L$ is the set

$$
W(L)=\left\{(L s, s):\|s\|_{2}=1\right\} .
$$

(iii) A related quantity is the numerical bound of $L$, defined by

$$
\omega(L)=\sup _{\lambda \in W(L)} \operatorname{Re} \lambda
$$

(iv) For $a>0$ and $R>0$ let $\mathrm{G}:=\{(a, R): \omega(L)>0, \sigma(L)<0\}$ denote the stability gap. Clearly, $\mathrm{G}=\left\{(a, R): a>0, R_{E}(a)<R<R_{L}(a)\right\}$.

An important property of the numerical range is that the spectrum of the operator $L$ is contained in the closure of its numerical range: i.e. $\Sigma(L) \subset \overline{W(L)}$, see for instance [15]. This implies that $\omega(L) \geqslant \sigma(L)$. 
From (LP), using Definition 3, we find the relation

$$
\frac{1}{2} \frac{\mathrm{d}}{\mathrm{d} t} \mathscr{E}(t)=\frac{(L s, s)}{\|s\|_{2}^{2}} \mathscr{E}(t) \leqslant \omega(L) \mathscr{E}(t) .
$$

The linear problem is stable with respect to $\|\cdot\|_{2}$ provided $\omega(L)<0$ and neutral stable when $\omega(L) \equiv 0$. The Euler-Lagrange equation for the maximum problem (30) is given by

$$
D^{2} s-a^{2} s+\frac{1}{2} R\left\{\mathrm{e}^{-z} B(s)+B\left(\mathrm{e}^{-z} s\right)\right\}=\omega s,
$$

which we solve for $s \in H_{0}^{1}\left(\mathbb{R}_{+}\right)$. Let $\omega_{\max }$ be the largest real eigenvalue of (31). Then $\omega(L)=\omega_{\max }$. Figure 3(A) shows the isocurves of $\omega_{\max }$. Observe that the zero-level curve corresponds to $R_{E}(a)$, see also Figure 1 .

A formal Taylor expansion of $\mathscr{E}(t)$ at $t=0$ gives

$$
\mathscr{E}(t)=\mathscr{E}(0)+\mathscr{E}^{\prime}(0) t+\mathrm{O}\left(t^{2}\right) .
$$

Now using (30), we have $\mathscr{E}^{\prime}(0) / \mathscr{E}(0)=2(L f, f) /\|f\|_{2}^{2}$, where $f$ is the initial condition given by $(\mathrm{LP})_{4}$. Let $\mu$ denote the maximal initial slope of $\mathscr{E}(t) / \mathscr{E}(0)$, i.e.

$$
\frac{1}{2} \mu=\sup _{\bar{f} \in H_{0}^{1}\left(\mathbb{R}_{+}\right)} \frac{(L \bar{f}, \bar{f})}{\|\bar{f}\|_{2}^{2}}=\omega(L) \quad \text { by Definition 3(ii, iii) . }
$$

The initial condition $f$ that maximizes the initial slope is found by solving the eigenvalue problem

$$
\frac{1}{2}\left(L+L^{\dagger}\right) \tilde{f}=\frac{1}{2} \mu \tilde{f} \quad \text { in } H_{0}^{1}\left(\mathbb{R}_{+}\right) .
$$

The 'energy' $\mathscr{E}(t)$ corresponding to the maximal initial growth $\tilde{f}(z)$ is shown in Figure 3(B), dashed curve. The computed evolution of the initial perturbation $\tilde{f}(z)$ is shown in Figure 4. The perturbation 'mass' is redistributed due to the existing upward throughflow, implying transient growth of $\mathscr{E}(t)$. Since the system is (linearly) stable, i.e. $\sigma(L)<0$, the perturbation will eventually decay to zero.

We conclude with some bounds on the growth of $\mathscr{E}(t)$. From $(\widehat{\mathrm{LP}})$ and using Definition 3(i) we obtain

$$
\|u(t)\|_{2}^{2} \leqslant \mathrm{e}^{2 \sigma(\widehat{L}) t}\|g\|_{2}^{2} .
$$

From (33), (16) and using the fact that $\sigma(\widehat{L})=\sigma(L)$, it follows that

$$
\|s(t)\|_{2}^{2} \leqslant\|u(t)\|_{2}^{2} \leqslant K \mathrm{e}^{2 \sigma(L) t}\|f\|_{2}^{2},
$$

where $K:=\|g\|_{2}^{2} /\|f\|_{2}^{2}$. Further, we have the estimate

$$
\|s(t)\|_{2}^{2} \leqslant \mathrm{e}^{2 \omega(L) t}\|f\|_{2}^{2},
$$




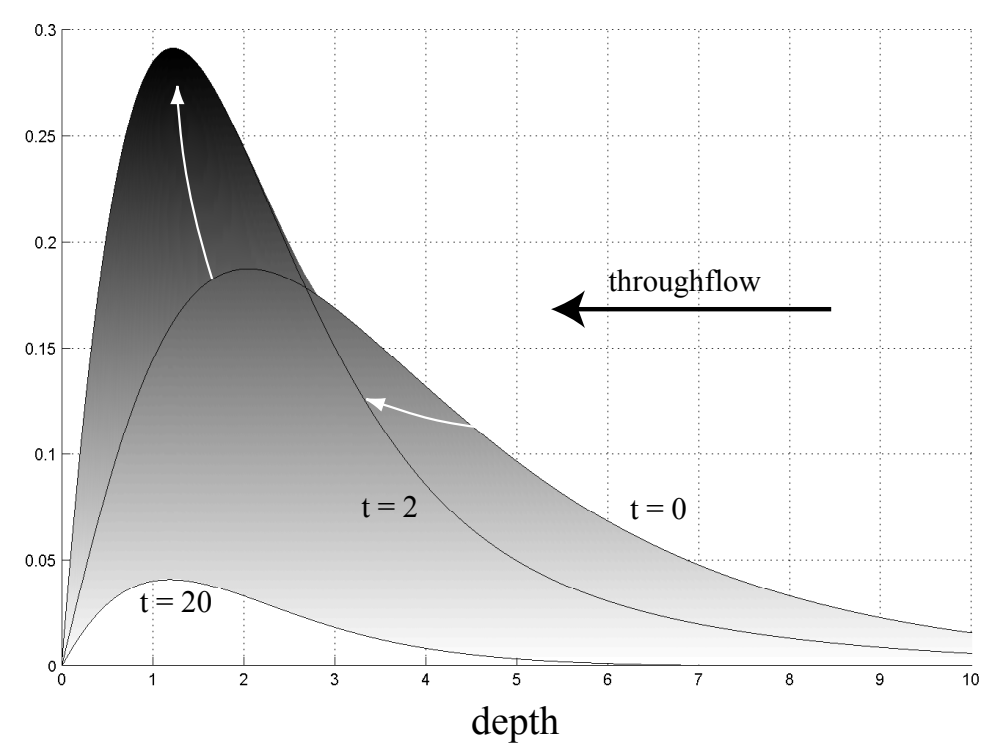

Fig. 4. Evolution of the solution of (LP) with initial perturbation $\tilde{f}(z)$. The corresponding functional $\mathscr{E}$ is depicted in Figure 3(B) for $\alpha=0$.

which follows directly from Definition 3. Combining these two estimates results in the upper bound

$$
\frac{\mathscr{E}(t)}{\mathscr{E}(0)} \leqslant \min _{t \geqslant 0}\left\{\mathrm{e}^{2 \omega(L) t}, K \mathrm{e}^{2 \sigma(L) t}\right\}
$$

see also the dashed curves in Figure 3(B). Note that this upperbound is not sharp.

\section{Elimination of transient growth by weighted norms}

In Section 3 we showed that the linear operator $L$ can be symmetrized by transformation (16). In this section we generalize this idea by defining

$$
s(z, t):=\mathrm{e}^{-\frac{1}{2} \alpha z} u(z, t), \quad \alpha \in[0,1] .
$$

Substitution of (35) in (LP) gives the problem

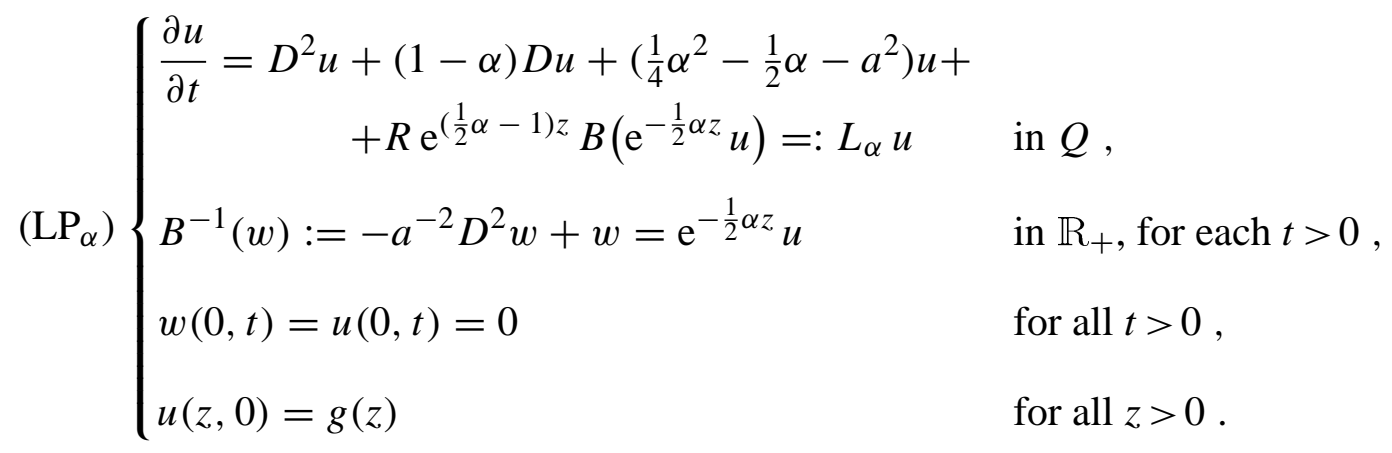

Note that $\mathrm{e}^{-\frac{1}{2} \alpha z} L_{\alpha} \mathrm{e}^{\frac{1}{2} \alpha z} \equiv L$, i.e. $L_{\alpha}$ is similar to $L$ for each $\alpha \in[0,1]$. 


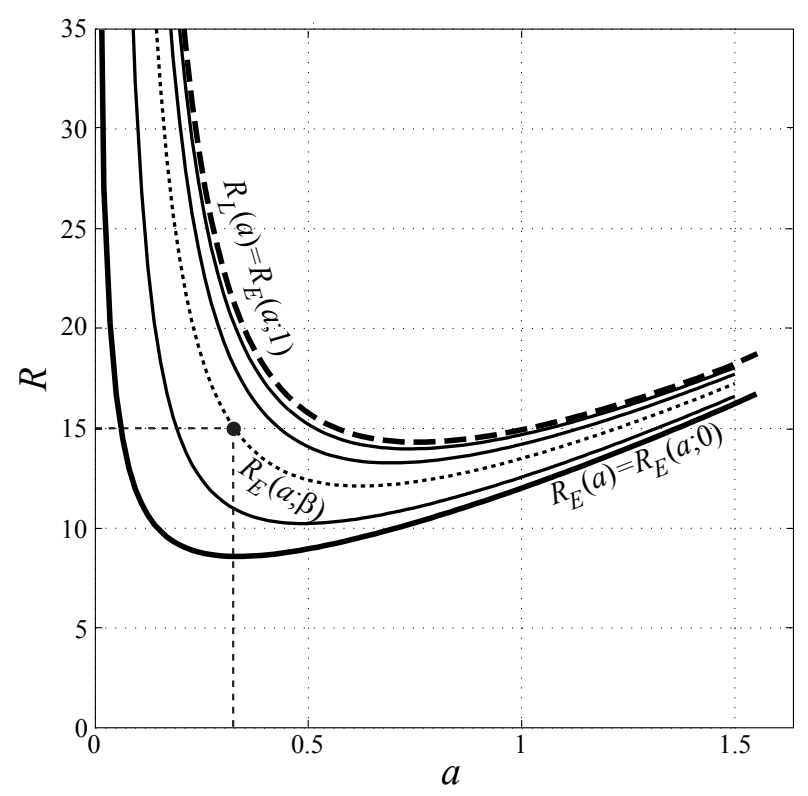

Fig. 5. Lowest eigenvalue $R_{E}(a ; \alpha)$, for various $\alpha \in[0,1]$, versus wavenumber $a>0$ for the equilibrium boundary layer (cf. Proposition 4). Bold solid curve $(\alpha=0)$ and bold dashed curve $(\alpha=1)$ are taken from Figure 1 . The dashed curve corresponds to $\alpha=\beta \in(0,1)$.

From this point on we redefine the energy functional. Let $u(z, t)$ be a solution of $\left(\mathrm{LP}_{\alpha}\right)$. Then

$$
\mathscr{E}(t):=\int_{\mathbb{R}_{+}} u^{2}(z, t) \mathrm{d} z=\int_{\mathbb{R}_{+}} s^{2}(z, t) \mathrm{e}^{\alpha z} \mathrm{~d} z,
$$

where $s(z, t)$ is the solution of (LP). We want to investigate the transient growth of solutions of $\left(\mathrm{LP}_{\alpha}\right)$ for $\alpha \in[0,1]$. We argue as in Section 4 to achieve monotonic decay of $\mathscr{E}(t)$ with respect to time. The variational formulation based on $L_{\alpha}$ yields the eigenvalue problem

$$
\begin{aligned}
D^{2} u+\left(\frac{1}{4} \alpha^{2}-\right. & \left.\frac{1}{2} \alpha-a^{2}\right) u= \\
& -\frac{1}{2} R\left\{\mathrm{e}^{\left(\frac{1}{2} \alpha-1\right) z} B\left(\mathrm{e}^{-\frac{1}{2} \alpha z} u\right)+\mathrm{e}^{-\frac{1}{2} \alpha z} B\left(\mathrm{e}^{\left(\frac{1}{2} \alpha-1\right) z} u\right)\right\}
\end{aligned}
$$

for $u \in H_{0}^{1}\left(\mathbb{R}_{+}\right)$. For given $a>0$ and $\alpha \in[0,1]$, let $R_{E}(a ; \alpha)$ be the smallest positive eigenvalue of (36). Observe that for $\alpha=1$ we regain eigenvalue problem (21) with $\sigma=0$, i.e. neutral stability. Therefore, $R_{E}(a ; 1)=R_{L}(a)$. For $\alpha=0$, eigenvalue problem (36) reduces to the one discussed in [33] and hence $R_{E}(a ; 0)=R_{E}(a)$. The stability curves $R=R(a ; \alpha)$ for $a>0$ and for fixed $\alpha \in[0,1]$ are shown in Figure 5, see also Figure 1. They are ordered in the following sense.

Proposition 4 For any $\alpha \in[0,1)$ we have $R_{E}(a ; \alpha)<R_{L}(a)$ for all $a>0$.

PROOF. Let $a>0$ be fixed and let $\left(s_{1}, w_{1}, R_{L}(a)\right)$ be the first eigensolution of the 
problem (in $\mathbb{R}_{+}$)

$$
\left\{\begin{array}{l}
D^{2} s_{1}+D s_{1}-a^{2} s_{1}=-R_{L}(a) \mathrm{e}^{-z} w_{1} \\
-D^{2} w_{1}+a^{2} w_{1}=a^{2} s_{1}
\end{array}\right.
$$

with $s, w \in H_{0}^{1}\left(\mathbb{R}_{+}\right)$. Next set $s_{1}=\mathrm{e}^{-\frac{1}{2} \alpha z} u_{1}$. Subsitution in (37) yields

$$
\left\{\begin{array}{l}
D^{2} u_{1}+(1-\alpha) D u_{1}+\left(\frac{1}{4} \alpha^{2}-\frac{1}{2} \alpha-a^{2}\right) u_{1}=-R_{L}(a) \mathrm{e}^{\left(\frac{1}{2} \alpha-1\right) z} w_{1} \\
-D^{2} w_{1}+a^{2} w_{1}=a^{2} u_{1} \mathrm{e}^{-\frac{1}{2} \alpha z}
\end{array}\right.
$$

Multiplying (38a) by $u_{1}$ and integrating over $\mathbb{R}_{+}$gives for $\alpha \in[0,1$ )

$$
\frac{1}{R_{L}(a)}=\frac{\left(\mathrm{e}^{\left(\frac{1}{2} \alpha-1\right) z} w_{1}, u_{1}\right)}{\left\|D u_{1}\right\|_{2}^{2}-\left(\frac{1}{4} \alpha^{2}-\frac{1}{2} \alpha-a^{2}\right)\left\|u_{1}\right\|_{2}^{2}} .
$$

From this we immediately derive

$$
\frac{1}{R_{E}(a ; \alpha)}>\frac{1}{R_{L}(a)}
$$

for all $a>0$.

The curves in Figure 5 have the following interpretation. The operator $L_{\alpha}$, defined in equation $\left(\mathrm{LP}_{\alpha}\right)_{1}$, is self-adjoint (and hence normal) for $\alpha=1$. For this particular case we find $R_{E}(a ; 1)=R_{L}(a)$ for all $a>0$, see Section 4. For $\alpha \in[0,1)$ we have $R_{E}(a ; \alpha)<R_{L}(a)$ for all $a>0$, i.e. there exists a stability gap. Now let the system Rayleigh number $R_{S}$ be such that for some $a>0$ it satisfies $R_{E}(a)<R_{S}<$ $R_{L}(a)$. Then there exists a unique $\beta \in(0,1)$ such that $R_{E}(a, \beta)=R_{s}$, see Figure 5, dotted curve. Then the described construction implies that for each $\alpha \in(\beta, 1)$ we have

$$
\frac{\mathrm{d}}{\mathrm{d} t} \int_{\mathbb{R}_{+}} s^{2}(z, t) \mathrm{e}^{\alpha z} \mathrm{~d} z<0 \quad \text { provided } \quad \int_{\mathbb{R}_{+}} f^{2}(z) \mathrm{e}^{\alpha z} \mathrm{~d} z<\infty .
$$

For fixed $(a, R) \in \mathrm{G}$ we solved (LP). The initial condition $f$ is chosen in such a way that $\int_{\mathbb{R}_{+}} f^{2}(z) \mathrm{e}^{z} \mathrm{~d} z<\infty$. The behaviour of $\mathscr{E}(t) / \mathscr{E}(0)$ is shown in Figure 3(B). The maximal initial growth of $\mathscr{E}(t) / \mathscr{E}(0)$ is obtained for $\alpha=0$. For increasing $\alpha$, the initial slope decreases and becomes negative. This is also to be expected since for some $\beta \in(0,1)$ the pair $(a, R)$ lies under the stability curve $R_{E}(a, \beta)$, i.e. the numerical bound becomes negative, see Figure 5. From this we conclude that $\int_{\mathbb{R}_{+}} s^{2}(z, t) \mathrm{e}^{\alpha z} \mathrm{~d} z$ exhibits transient growth for $\alpha \in(0, \beta)$. 


\section{Discussion}

In [33] we introduced and studied gravitational instability of a saline boundary layer formed by evaporation induced upward throughflow at the horizontal surface of a porous medium. In that study several important questions remained untouched. The purpose of the present paper is to resolve these questions. In particular, we present a complete picture of the spectrum of the linearised problem and we prove exchange of stabilities by introducing a similar self-adjoint operator.

Because of the evaporation induced convection, the linearised perturbation equations are non-self-adjoint. This results in the appearance of a stability gap, i.e. the linear instability bound does not coincide with the energy stability bound. By using again similar operators, and thereby introducing weighted norms in the energy method, we show that these bounds coincide for small perturbations.

The stability gap suggests the existence of subcritical finite amplitude solutions, i.e. nontrivial solutions that exist for Rayleigh numbers below the critical linear instability threshold. The existence of such solutions is shown by Pieters and Schuttelaars, [22]. These authors describe a fairly complete bifurcation analysis of the problem discussed in this paper. The existence of subcritical solutions, however, does not contradict the coinciding stability thresholds since we do not incorporate finite amplitude nonlinear interactions in our approach.

\section{A Appendix : A modified Chebyshev-Galerkin method}

In this appendix we discretize problem $\left(\operatorname{LP}_{\alpha}\right)$ for $\alpha \in[0,1]$. We use the method of lines, considering space and time discretisations separately. For the spatial discretisation we use a modified Chebyshev-Galerkin method. Essentially, there are two approaches to discretize $L_{\alpha}$ on a semi-infinite domain:

(1) truncation of the semi-infinite domain $\mathbb{R}_{+}$to the finite interval $(0, h),[19,34]$,

(2) transformation of $\mathbb{R}_{+}$to the finite interval $(0, h)$ by virtue of an algebraic mapping, [9], or an exponential mapping, [30].

Option (2) results in a modified operator $L_{\alpha}$ in which the derivatives are multiplied by additional coefficients which depend on the used mapping and vary in space. In particular, these transformations bring in singular coefficients in the operator and this strongly influences the efficiency of the method. Therefore we choose here for option (1). For the domain truncation we need an additional boundary condition at $z=h$. For initial conditions satisfying $g \in H_{0}^{1}\left(\mathbb{R}_{+}\right) \cap L^{\infty}\left(\mathbb{R}_{+}\right)$, we have $u \rightarrow 0$ as $z \rightarrow \infty$, see [21] for details. Based on this observation, it is reasonable to impose a zero Dirichlet condition at $z=h$.

The standard Chebyshev-Galerkin method uses the Chebyshev polynomials for both the shape and the test functions. This leads in general to full matrices, which is numerically inefficient. The modification of the standard Chebyshev-Galerkin method is basically a combination of ideas posed by Heinrichs, [11, 12], Shen, [29], and also Pop, [23]. Key feature in Galerkin methods is the use of shape and test functions that satisfy a priori the boundary conditions. However, there are many 
ways to put the boundary conditions in the shape and test functions. In this appendix they are chosen in such a way that the differentiation operators $\left(D^{2}, D\right)$ lead to banded matrices having good condition numbers, which makes the numerical treatment more efficient. Further, the technique followed in this appendix also removes so-called spurious eigenvalues.

To use the Chebyshev-Galerkin method, we need to transform problem $\left(\mathrm{LP}_{\alpha}\right)$ from the truncated interval $(0, h)$ to the interval $(-1,1)$. These operations can be summarized in the linear transformation $\zeta:(0, h) \longmapsto(-1,1)$, defined by $\zeta=$ $\frac{2}{h} z-1$, or $z=\frac{1}{2} h(\zeta+1)$. Let $u(z)=u\left(\frac{1}{2} h(\zeta+1)\right)=\bar{u}(\zeta)$ and $w(z)=w\left(\frac{1}{2} h(\zeta+1)\right)=$ $\bar{w}(\zeta)$. Then substitution in $\left(\mathrm{LP}_{\alpha}\right)$ yields

$$
\left\{\begin{aligned}
\frac{\partial \bar{u}}{\partial t}=\ell^{2} \bar{D}^{2} \bar{u}+\ell(1-\alpha) \bar{D} \bar{u}+ & \left(\frac{1}{4} \alpha^{2}-\frac{1}{2} \alpha-a^{2}\right) \bar{u}+ \\
& +R \mathrm{e}^{\left(\frac{1}{2} \alpha-1\right) z(\zeta)} \bar{B}\left(\mathrm{e}^{-\frac{1}{2} \alpha z(\zeta)} \bar{u}\right), \\
\bar{B}^{-1} \bar{w}=-a^{-2} \ell^{2} \bar{D}^{2} \bar{w}+\bar{w}= & \mathrm{e}^{-\frac{1}{2} \alpha z(\zeta)} \bar{u},
\end{aligned}\right.
$$

where $\ell=\frac{2}{h}$. From this point on we drop the bars. Next, the unknown functions $\{u, w\}$ are approximated by

$$
\{u, w\}(\zeta, t)=\sum_{j=0}^{\infty}\left\{u_{j}, w_{j}\right\}(t) \mathcal{W}_{j}(\zeta)
$$

The initial space is approximated with finite dimensional ones. Therefore, a truncated series up to an order $K$ is considered. The result is projected on a finite dimensional space in order to get a finite algebraic system. Let $T_{k}(\zeta)$ denote the Chebyshev polynomial of degree $k$. We define the shape functions $([11,12])$

$$
\mathcal{W}_{k}(\zeta)=\left(1-\zeta^{2}\right) T_{k}(\zeta), \quad k \geqslant 0
$$

and approximate $\{u, w\}$ by $\left\{u_{K}, w_{K}\right\}=\sum_{j=0}^{K}\left\{u_{j}, w_{j}\right\} \mathcal{W}_{j}$. Clearly,

$$
\mathbb{X}_{K}=\operatorname{span}\left\{\mathcal{W}_{k}, k=\overline{0, K}\right\}=\left\{p \in \mathbb{P}_{K+2}: p( \pm 1)=0\right\}
$$

and therefore $\left\{u_{K}, w_{K}\right\}$ satisfy the boundary conditions a priori. For the definition of the test functions we use (cf. [8, 29])

$$
\mathcal{V}_{k}(\zeta)=T_{k}(\zeta)-T_{k+2}(\zeta), \quad k \geqslant 0
$$

Obviously,

$$
\mathbb{Y}_{K}=\operatorname{span}\left\{\mathcal{V}_{k}, k=\overline{0, K}\right\}=\left\{p \in \mathbb{P}_{K+2}: p( \pm 1)=0\right\}
$$


We find the following semi-discretisation

$$
\left\{\begin{array}{l}
\mathbf{I} \frac{\mathrm{d} \mathbf{u}}{\mathrm{d} t}=\mathbf{D}_{2} \mathbf{u}+(1-\alpha) \mathbf{D}_{1} \mathbf{u}+\left(\frac{1}{4} \alpha-\frac{1}{2} \alpha-a^{2}\right) \mathbf{I} \mathbf{u}+R \mathbf{E}_{2} \mathbf{w} \\
\mathbf{B}^{-1} \mathbf{w}:=-a^{-2} \mathbf{D}_{2} \mathbf{w}+\mathbf{I} \mathbf{w}=\mathbf{E}_{1} \mathbf{u} \\
\mathbf{u}(0)=\mathbf{u}^{0}
\end{array}\right.
$$

The matrices $\mathbf{I}, \mathbf{D}_{1}, \mathbf{D}_{2}, \mathbf{E}_{1}$ and $\mathbf{E}_{2}$ are defined as follows:

$$
\begin{aligned}
& \mathbf{I}_{p, k}=\left(\mathcal{W}_{p}, \mathcal{V}_{k}\right)_{\omega}, \\
& \mathbf{D}_{1_{p, k}}=\left(\ell D \mathcal{W}_{p}, \mathcal{V}_{k}\right)_{\omega}, \quad \mathbf{D}_{2_{p, k}}=\left(\ell^{2} D^{2} \mathcal{W}_{p}, \mathcal{V}_{k}\right)_{\omega}, \\
& \mathbf{E}_{1_{p, k}}=\left(\mathrm{e}^{-\frac{1}{2} \alpha z(\zeta)} \mathcal{W}_{p}, \mathcal{V}_{k}\right)_{\omega}, \quad \mathbf{E}_{2_{p, k}}=\left(\mathrm{e}^{\left(\frac{1}{2} \alpha-1\right) z(\zeta)} \mathcal{W}_{p}, \mathcal{V}_{k}\right)_{\omega},
\end{aligned}
$$

where $(u, v)_{\omega}=\int_{-1}^{1} u v \omega \mathrm{d} \zeta$ and $\omega(\zeta)=\left(1-\zeta^{2}\right)^{-1 / 2}$. The matrices $\mathbf{I}, \mathbf{D}_{1}$, and $\mathbf{D}_{2}$ can be determined explicitly. Table A shows the coefficients. Matrices $\mathbf{E}_{1}$ and $\mathbf{E}_{2}$ are determined by a Gauss-Lobatto integration method ([24]), i.e.

$$
\mathbf{E}_{1_{p, k}} \cong \sum_{j=0}^{(p+2)(k+2)+1} \beta \mathrm{e}^{-\frac{1}{2} \alpha z\left(\zeta_{j}\right)} \mathcal{W}_{p}\left(\zeta_{j}\right) \mathcal{V}_{k}\left(\zeta_{j}\right)
$$

and

$$
\mathbf{E}_{2_{p, k}} \cong \sum_{j=0}^{(p+2)(k+2)+1} \beta \mathrm{e}^{\left(\frac{1}{2} \alpha-1\right) z\left(\zeta_{j}\right)} \mathcal{W}_{p}\left(\zeta_{j}\right) \mathcal{V}_{k}\left(\zeta_{j}\right)
$$

where

$$
\zeta_{j}:=-\cos \frac{(2 j+1) \pi}{2((p+2)(k+2)+1)}, \quad \beta:=\frac{\pi}{(p+2)(k+2)+1}
$$

are respectively the Gauss nodes and the corresponding (constant) weighting coefficients.

Let $\mathbf{u}^{n}$ be the solution of (A.3) at time $t=n \cdot \Delta t$ and let $\mathbf{A}:=\mathbf{D}_{2}+(1-\alpha) \mathbf{D}_{1}+$ $\left(\frac{1}{4} \alpha^{2}-\frac{1}{2} \alpha-a^{2}\right) \mathbf{I}$. Discretization in time is given by

$$
\begin{aligned}
\mathbf{u}^{0} & =\mathbf{u}^{0}, \\
\mathbf{w}^{0} & =\mathbf{B E}_{1} \mathbf{u}^{0} \\
(\mathbf{I}-\Delta t \mathbf{A}) \mathbf{u}^{n+1} & =\mathbf{u}^{n}-\Delta t R \mathbf{E}_{2} \mathbf{w}^{n} \\
\mathbf{w}^{n+1} & =\mathbf{B E}_{1} \mathbf{u}^{n+1} .
\end{aligned}
$$


Table A.1

Discretization matrices. Here $c_{p}=0$ for $p<0, c_{p}=2$ for $p=0$ and $c_{p}=1$ for $p>0$. All coefficients have to be multiplied by $\frac{1}{2} \pi$.

\begin{tabular}{|c|c|c|c|}
\hline & $k=p-4$ & $k=p-3$ & $k=p-2$ \\
\hline $\mathbf{I}_{p, k}=\left(\mathcal{W}_{p}, \mathcal{V}_{k}\right)_{\omega}$ & $\frac{1}{4}$ & & $-\frac{1}{2}-\frac{1}{4} c_{p-2}$ \\
\hline $\mathbf{D}_{2_{p, k}}=\left(\ell^{2} D^{2} \mathcal{W}_{p}, \mathcal{V}_{k}\right)_{\omega}$ & & & $\ell^{2}\left(p(p-3)+2 c_{p}\right)$ \\
\hline $\mathbf{D}_{1_{p, k}}=\left(\ell D \mathcal{W}_{p}, \mathcal{V}_{k}\right)_{\omega}$ & & $\ell\left(1-\frac{1}{2} p\right)$ & \\
\hline
\end{tabular}

\begin{tabular}{|c|c|c|c|c|}
\hline & $k=p-1$ & $k=p$ & $k=p+1$ & $k=p+2$ \\
\hline$\left(\mathcal{W}_{p}, \mathcal{V}_{k}\right)_{\omega}$ & & $\frac{1}{2}+\frac{1}{2} c_{p}-\frac{1}{4} c_{p-1}$ & & $-\frac{1}{4} c_{p}$ \\
\hline$\left(\ell^{2} D^{2} \mathcal{W}_{p}, \mathcal{V}_{k}\right)_{\omega}$ & & $\ell^{2}\left(-p(p+3)-2 c_{p}\right)$ & & \\
\hline$\left(\ell D \mathcal{W}_{p}, \mathcal{V}_{k}\right)_{\omega}$ & $\ell\left(p+\frac{1}{2}\left(c_{p}-c_{p-1}\right)\right)$ & & $\ell\left(-c_{p}-\frac{1}{2} p\right)$ & \\
\hline
\end{tabular}

\section{References}

[1] W. O. Criminale, T. L. Jackson, and R. D. Joslin. Theory and Computation of Hydrodynamic Stability. Cambridge University Press, Cambridge, 2003.

[2] S. H. Davis. Buoyancy, surface tension in stability by the method of energy. J. Fluid Mech., 39:347, 1969.

[3] S. H. Davis. On the principle of exchange of instabilities. Proc. Roy. Soc. Ser. A, 310:341-358, 1969.

[4] S. H. Davis. On the possibility of subcritical instabilities. In Instability of Continuous Systems, Proc. IUTAM Sympos. Herrenalb, pages 222-227, Berlin, 1971. Springer-Verlag.

[5] B. F. Farrell and P. J. Ioannou. Generalized stability theory. Part I: Autonomous operators. J. Atmos. Sci., 53(14):2025-2040, 1996.

[6] G. P. Galdi and M. Padula. A new approach to energy theory in the stability of fluid motion. Arch. Ration. Mech. Anal., 110(3):187-286, 1990.

[7] G. P. Galdi and B. Straughan. Exchange of stabilities, symmetry, and nonlinear stability. Arch. Rat. Mech. Anal., 89:211-228, 1985.

[8] D. Gottlieb and S. A. Orszag. Numerical Analysis of Spectral Methods: The- 
ory and Applications. SIAM, Philadelphia, 1983.

[9] C. E. Grosch and S. A. Orszag. Numerical solution of problems in unbounded regions: Coordinate transforms. J. Comp. Phys., 25:273-296, 1977.

[10] C. E. Grosch and H. Salwen. The continuous spectrum of the OrrSommerfeld equation. part 1 . the spectrum and the eigenfunctions. J. Fluid Mech., 87:33-54, 1978.

[11] W. Heinrichs. Improved condition number for spectral methods. Math. Comput., 53:103-119, 1989.

[12] W. Heinrichs. Stabilization techniques for spectral methods. J. Sci. Comp., 6:1-19, 1991.

[13] G. M. Homsy and A. E. Sherwood. Convective instabilities in porous media with throughflow. Technical Report UCRL-76539, Lawrence Livermore Lab. Rep., 1975.

[14] G. M. Homsy and A. E. Sherwood. Convective instabilities in porous media with throughflow. Amer. Inst. Chem. Engrs. J., 22:168-174, 1976.

[15] V. Istratescu. Introduction to Linear Operators. Marcel-Dekker, New York, 1981.

[16] T. Kato. Perturbation Theory for Linear Operators. Springer-Verlag, New York, 1976.

[17] E. R. Lapwood. Convection of a fluid in a porous medium. Proc. Cambridge Phil. Soc., 44:508-521, 1948.

[18] D. G. Lasseigne, R. D. Joslin, T. L. Jackson, and W. O. Criminale. The transient period for boundary layer disturbances. J. Fluid Mech., 381:89-119, 1999.

[19] L. M. Mack. A numerical study of the temporal eigenvalue spectrum of the Blasius boundary layer. J. Fluid Mech., 73:497-520, 1976.

[20] D. A. Nield and A. Bejan. Convection in Porous Media. Springer-Verlag, New York, 2nd edition, 1992.

[21] G. J. M. Pieters, I. S. Pop, and C. J. van Duijn. A note on the solution of a coupled parabolic-elliptic system arising in linear stability analysis of gravitydriven porous media flow. Technical Report CASA 04-30, Eindhoven University of Technology, 2004. (submitted to ZAMP).

[22] G. J. M. Pieters and H. M. Schuttelaars. Bifurcation analysis for the steady boundary layer formed by throughflow near the surface of a porous medium. (in preparation).

[23] I. S. Pop. Numerical approximation of differential equations by spectral methods. Technical report, "Babes-Bolyai" University, Cluj-Napoca, 1995. (in romanian).

[24] A. Quarteroni, R. Sacco, and F. Saleri. Numerical Mathematics. SpringerVerlag, New York, 2000.

[25] S. C. Reddy and D. S. Henningson. Energy growth in viscous channel flows. J. Fluid Mech., 252:209-238, 1993.

[26] S. C. Reddy and L. N. Trefethen. Pseudospectra of the convection-diffusion operator. SIAM J. Appl. Math., 54:1634-1649, 1994.

[27] H. Salwen and C. E. Grosch. The continuous spectrum of the Orr- 
Sommerfeld equation. part 2. eigenfunction expansions. J. Fluid Mech., 104:445-465, 1981.

[28] P. J. Schmidt and D. S. Henningson. Stability and Transition in Shear Flows. Springer, Berlin, 2001.

[29] J. Shen. Efficient spectral-Galerkin method II. Direct solvers of second and fourth order equations by using Chebyshev polynomials. SIAM J. Sci. Comput., 16:74-87, 1995.

[30] P. R. Spalart. A spectral method for external viscous flows. Contemp. Math., 28:315-335, 1984.

[31] B. Straughan. The Energy Method, Stability and Nonlinear Convection, volume 91 of Applied Mathematical Sciences. Springer-Verlag, New-York, 2nd edition, 2003.

[32] C. J. Van Duijn, G. J. M. Pieters, and P. A. C. Raats. Steady flows in unsaturated soils are stable. Trans. Porous Media, 57:215-244, 2004.

[33] C. J. Van Duijn, G. J. M. Pieters, R. A. Wooding, and A. van der Ploeg. Stability criteria for the vertical boundary layer formed by throughflow near the surface of a porous medium. In P. A. C. Raats, D. Smiles, and A. W. Warrick, editors, Environmental Mechanics - Water, Mass and Energy Transfer in the Biosphere - The Philip Volume, volume 129 of Geophysical Monographs, pages 155-169. American Geophysical Union, 2002.

[34] T. L. Van Stijn and A. I. Van de Vooren. An accurate method for solving the Orr-Sommerfeld equation. J. Eng. Math., 14:17-26, 1980.

[35] R. A. Wooding, S. W. Tyler, and I. White. Convection in groundwater below an evaporating salt lake: 1. Onset of instability. Water Resour. Res., 33:11991217, 1997.

[36] R. A. Wooding, S. W. Tyler, I. White, and P. A. Anderson. Convection in groundwater below an evaporating salt lake: 2. Evolution of fingers or plumes. Water Resour. Res., 33(6):1219-1228, 1997. 